\title{
Propiedades psicométricas de una versión informatizada del Physical Self Description Questionnaire en adolescentes mexicanos Psychometric properties of a computerized version of the Physical Self-Description Questionnaire in Mexican adolescents \\ *Martha Ornelas Contreras, *Miguel Conchas Ramírez, **Ana Citlalli Díaz-Leal, **Luis Humberto Blanco Ornelas, *Yunuen Socorro Rangel Ledezma \\ *Universidad Autónoma de Chihuahua (México), **Universidad de Guadalajara (México)
}

Resumen. El objetivo de esta investigación fue determinar las propiedades psicométricas propuestas por Marsh, Martin y Jackson (2010) para la versión corta del Physical Self Description Questionnaire (PSDQ-S). La muestra total fue de 1071 adolescentes de la ciudad de Chihuahua, México, con edades comprendidas entre 13 y 18 años ( $M=15.52 \pm 1.42$ años). La aplicación fue de forma sistematizada. La estructura factorial del cuestionario se realizó mediante análisis factoriales exploratorios y confirmatorios. Éstos, mostraron una estructura de nueve factores: actividad física, apariencia, grasa corporal, coordinación, resistencia, flexibilidad, salud, competencia deportiva y fuerza, los cuales demostraron que el cuestionario es válido y confiable (GFI=.956; $R M S E A=.041 ; C F I=.975)$. La estructura de los nueve factores atiende a criterios estadísticos y sustantivos, ha mostrado adecuados indicadores de ajuste de fiabilidad y validez. En conclusión, el PSDQ-S puede ser una herramienta útil para avanzar en el estudio de los factores que afectan al autoconcepto físico en adolescentes.

Palabras clave: validez; fiabilidad; psicometría; evaluación psicológica; autoconcepto físico.

Abstract. The aim of this research was to determine the psychometric properties proposed by Marsh et al. (2010) for the Physical SelfDescription Questionnaire (PSDQ-S). The total sample has been composed by 1,071 teenagers from the city of Chihuahua, México, with ages from 13 to 18 years old $(M=15.52 \pm 1.42$ years). The Questionnaire was by computer. The factor structure of the questionnaire was analyzed through confirmatory factor analysis. This analysis shows that a nine-factor structure is viable and adequate for the total sample and the populations of athletes and non-athletes. The nine-factor structure (physical activity, appearance, body fat, coordination, endurance, flexibility, health, sport competence, and strength) is viable and reliable (GFI=.956; $R M S E A=.041 ; C F I=.975$ ). According to statistical and substantive criteria, the nine-factor structure showed adequate indicators of reliability and validity adjustments. In conclusion, the PSDQ-S can be a useful tool to advance in the study of the factors affecting physical self-concept.

Keywords: validity; reliability; psychometrics; psychological assessment; physical self-concept.

\section{Introducción}

Existe una preocupación por parte de la comunidad científica y profesionales de la salud por conocer el autoconcepto físico, debido a que la obesidad genera problemas de salud, hasta llegar a la muerte. En niños y adolescentes desde cinco a 19 años, contaban con sobrepeso u obesidad 340 millones en el 2016, con una prevalencia de 18\% (Organización Mundial de la Salud, 2018a). En México para el 2012 casi 4 de cada 10 adolescentes (12 a 19 años) presentan sobrepeso u obesidad con una prevalencia de 36.3\% (ENSANUT, 2016). Con estos datos al alza, es necesario hacer consciente a la población en conocerse, identificar los ideales si son posibles, con ello realizar hábitos saludables.

En la sociedad el autoconcepto es base importante para una adecuada interacción entre las personas, es multifacético, por el valor personal. En la presente investigación es de interés el autoconcepto físico como una parte del autoconcepto general. Éste consiste en la autopercepción de características físicas como la condición, competencia física o deportiva, coordinación, flexibilidad, imagen corporal, resistencia, fuerza, autoestima, potencia aeróbica (Fernández-Bustos, González-Martí, Contreras \& Cueva, 2015; Fernández, Contreras, García \& González, 2010; GraoCruces, Fernández-Martínez \& Nuviala, 2016; Moreno,

Fecha recepción: 03-12-12. Fecha de aceptación: 19-06-19 Yunuen Socorro Rangel Ledezma yrangel@uach.mx
Cervelló \& Moreno, 2008). Esta autopercepción varía según características físicas que presenta la persona como sobre peso, bajo peso o peso normal (Baena, Vega \& Ramírez, 2018), así como insatisfacción corporal y hábitos saludables que se adquieren desde edades tempranas (Longmuiremail, Colley, Wherley \& Tremblay, 2014).

La práctica de actividad física ayuda en la percepción de las capacidades y percepciones de autoconcepto (Kyle, Mendo, Garrido \& Sánchez, 2016; Martínez \& GonzálezHernández, 2018; Rosa, García, \& Carrillo, 2019), a su vez, visualizarse con fuerza muscular y peso adecuado, las personas se perciben resistentes, fuertes y satisfechos con su propio cuerpo, lo que contribuye a una adecuada autoestima. Lo anterior genera motivación para cumplir sus objetivos sobre autoconcepto físico, y continuar con la realización de actividad física

Las mujeres, ante los estereotipos presentan mayor preocupación por ser juzgadas por su apariencia por el uso de ropa, es específicamente deportiva (Baena et al., 2018). Es decir, una condición física saludable se asocia con un autoconcepto físico positivo (Grao-Cruces. et al., 2016; Reigal, Videra, Parra \& Juárez, 2012) y un auto concepto negativo genera vulnerabilidad ante la presión cultural sobre la delgadez (Rodríguez, González-Fernández \& Goñi, 2013).

El autoconcepto físico es generador de preocupación y cambios en la percepción de sí mismos en la adolescencia, de la imagen ideal y la real (Fernández-Bustos et al., 2015), debido a los cambios biológicos, físicos, psicológicos y sociales, así como los hábitos adquiridos para la vida adulta (McMichan, Gibson \& Rowe, 2018; Pakarinen, Parisod, Smed 
\& Salanterä, 2017), aunados con factores demográficos, sociológicos, conductuales educacionales y tradicionales (Moreno, et al., 2008), rendimiento académico (Yáñez, Barraza \& Mahecha, 2016), habilidades motrices (Murgui, García \& García, 2016). Se manifiestan consecuencias psicológicas como autoestima baja (Delgado-Floody, Carter-Thuillier, JerezMayorga, Cofré-Lizama \& Martínez-Salazar, 2018), depresión y trastornos alimenticios (Baile y, Hetrick, Rosenbaum, Purcell \& Parker, 2018).

En adolescentes entre 15 y 19 años la depresión es la tecer causa principal de enfermedad; entre 10 a 19 años, a nivel mundial uno de cada seis presentan sobrepeso u obseidad en el 2016, con un 20\% más en occidente que en oriente (Organización Mundial de la Salud, 2018b).

Por ello en el presente estudio (Montero \& León, 2005) se investiga la estructura factorial de la versión corta del Physical Self Description Questionnaire (PSDQ-S) propuesta por Marsh, Martín y Jackson, (2010), lo que se justifica por la importancia de comprobar la estructura factorial de un instrumento y la equivalencia psicométrica del mismo en distintos grupos (Abalo, Lévy, Rial \& Varela, 2006). Con la finalidad de validar en población mexicana esta versión.

El cuestionario de autoconcepto físico (PSDQ) es un instrumento multidimensional, las escalas han presentado cambios hasta crearse la versión corta. En un inicio presentaba once escalas de manera directa: Fuerza, Grasa Corporal, Actividad, Resistencia / Aptitud, Competencia Deportiva, Coordinación, Salud, Apariencia, Flexibilidad, Físico Global y Autoestima Global (Marsh, 1996; Marsh, Richards, Johnson, Roche y Tremayne, 1994). De los 70 ítems de la versión original, se generó una versión corta (PSDQ-S) con solo 40 ítems con resultados similares (Marsh, et al., 2010). Se realizó un estudio con anterioridad del autoconcepto con el physical self-concept Questionaire (Blanco, Banco, Viciana \& Zueck, 2015), pero dirigido a estudiantes universitarios mexicanos, se sugiere tener posibilidades de instrumentos válidos y confiables para diferentes poblaciones.

\section{Método}

\section{Participantes}

La muestra consta de 1071 adolescentes, todos ellos estudiantes de secundaria y nivel medio superior de la ciudad de Chihuahua, México, se obtuvo mediante un muestreo por conveniencia. La edad de los participantes fluctuó entre los 13 y 18 años, con una media de 15.52 y una desviación estándar de 1.42 años.

\section{Instrumento}

La versión corta del Physical Self Description Questionnaire (PSDQ-S) de Marsh et al. (2010), fue el cuestionario utilizado, éste consta de 40 ítems que evalúa el autoconcepto físico, distribuidos en once subescalas: apariencia, grasa corporal, coordinación, resistencia, flexibilidad, salud, actividad física, competencia deportiva, fuerza, autoconcepto físico general y autoestima. Las primeras nueve subescalas evalúan dimensiones específicas del autoconcepto físico, mientras que las dos últimas evalúan dimensiones generales del autoconcepto físico.

El participante responde a cada uno de los 40 ítems, en una escala de 1 (falso) a 6 (verdadero), según se encuentra de acuerdo con lo mencionado en cada uno de los ítems.

Para el presente estudio, además de traducir los ítems del cuestionario, se hicieron dos adaptaciones a la versión de Marsh et al. (2010). Motivo por el cual, se referirá al cuestionario PSDQ-S como cuestionario PSDQ-SM.

Dentro de la primera adaptación, en la versión original las seis opciones de respuesta se puntúan 1 (Falso) a 6 (Verdadero); esto fue modificada para la presente investigación, las opciones de respuesta van de 0 (Totalmente Falso) a 5 (Totalmente Verdadero). Esta se justifica porque las personas asocian con mayor naturalidad al «0» con la categoría «Totalmente Falso».

La segunda adaptación consistió en aplicar el instrumento por medio de una computadora con el fin de permitir el almacenamiento de los datos sin etapas previas de codificación, con una mayor precisión y rapidez.

\section{Procedimiento}

Una vez conseguido el permiso tanto de las autoridades educativas como el de las familias, se invitó a participar a estudiantes de secundaria y bachillerato de la ciudad de Chihuahua, México. Posteriormente, el instrumento fue aplicado en una sesión de 60 minutos; en las aulas de los centros educativos correspondientes. Al inicio de cada aplicación se dieron a conocer, a cada participante, los objetivos de la investigación y solicitaba su mayor sinceridad al contestar.

Una vez aplicado el instrumento se procedió a recopilar y analizar los datos mediante el paquete estadístico SPSS 18.0 y AMOS 21.0.

\section{Análisis de datos}

El primer paso consistió en calcular la asimetría y la curtosis de cada ítem, para determinar si se cumplía el supuesto de normalidad.

Luego, se sometieron a prueba dos modelos de medida: el PSDQ-SMA, que responde a una estructura de once factores acorde a la distribución original de los ítems dentro del cuestionario y el modelo PSDQ-SMB, correspondiente a la estructura factorial del modelo anterior sin los factores autoconcepto físico general y autoestima (por medir dimensiones generales del autoconcepto físico), así como los ítems que de acuerdo con los índices de modificación resultaron no adecuados.

Posteriormente se calculó la fiabilidad de los factores del mejor de los modelos de medida obtenido, a través del Coeficiente Alpha de Cronbach (Elosua \& Zumbo, 2008; Nunnally \& Bernstein, 1995) y del Coeficiente Omega (Revelle \& Zinbarg, 2009; Sijtsma, 2009).

Para conducir los análisis factoriales confirmatorios se utilizó el software AMOS 21 (Arbuckle, 2012), las varianzas de los términos de error fueron especificados como parámetros libres, en las variables latentes (factores) se fijó uno de los coeficientes estructurales asociados a uno, para que su escala sea igual a la de una de las variables observables (ítems). El método de estimación empleado fue el de Máxima Verosimilitud (ML) con la aplicación de procedimientos de remuestreo bootstrap para los casos de no normalidad (Byrne, 2010; Kline, 2011); aun cuando en AMOS 21.0 el ML es espe- 
cialmente robusto para posibles casos de no normalidad, especialmente si la muestra es suficientemente amplia y los valores de asimetría y curtosis no son extremos (asimetría < |2| y curtosis $<|7|)$.

Se siguió la recomendación de Thompson (2004), debido a que cuando se emplea análisis factorial confirmatorio se debe corroborar no sólo el ajuste de un modelo teórico, sino que es recomendable comparar los índices de ajuste de varios modelos alternativos para seleccionar el mejor.

Para evaluar el ajuste del modelo se emplearon el estadístico Chi-cuadrado, el índice de bondad de ajuste (GFI) y el error cuadrático medio de aproximación (RMSEA) como medidas absolutas de ajuste. El índice de bondad ajustado (AGFI), el Índice Tucker-Lewis (TLI) y el índice de bondad de ajuste comparativo (CFI) como medidas de ajuste incremental. La razón de Chi-cuadrado sobre los grados de libertad (CMIN/GL) y el Criterio de Información de Akaike (AIC) como medidas de ajuste de parsimonia (Byrne, 2010; Gelabert, et al., 2011).

\section{Resultados}

\section{Asimetría y curtosis de los ítems del cuestionario}

Todos los ítems muestran valores de asimetría de \pm 2.35 y \pm 4.90 de curtosis, por lo que se infiere que se ajustan razonablemente a una distribución normal; por otro lado, el índice multivariado de Mardia por encima del valor 70 indica ausencia de normalidad multivariada (Rodríguez \& Ruiz, 2008).

\section{Análisis factorial confirmatorio muestra total}

Los resultados globales del análisis factorial confirmatorio (GFI=.857; $R M S E A=.057$; $C F I=.914$ ) para el modelo PSDQSMA que corresponde a una estructura de once factores acorde a la distribución original de los ítems dentro del cuestionario, indican que el modelo de medición no es aceptable (Tabla 1).

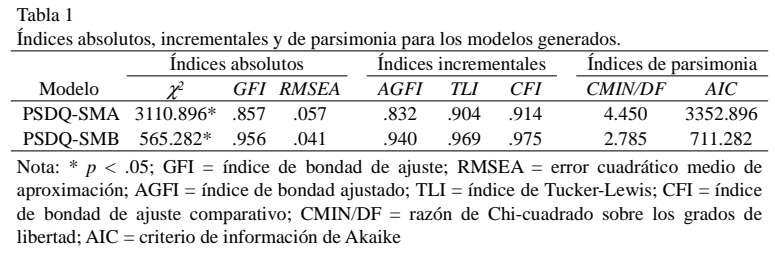

Los factores del modelo PSDQ-SMA explican aproximadamente el 73\% de la varianza y de acuerdo con los resultados obtenidos 9 de los 40 ítems saturan por debajo de .70 en su dimensión prevista (Tabla 2).

Los resultados globales del análisis factorial confirmatorio (GFI=.956; $R M S E A=.041 ; C F I=.975$ ) del segundo modelo sometido a prueba (PSDQ-SMB), corresponde a la estructura factorial del modelo anterior, pero sin los factores autoconcepto físico general y autoestima sin los ítems 1, 4, 7, 8, 12, 13, 15, 32 y 36 indican que este modelo de medición es mejor que el modelo anterior y que su ajuste es óptimo (Tabla 1). Los factores de este modelo explican aproximadamente el $80 \%$ de la varianza. Por otro lado solo dos de los ítems satura por debajo de .70 en su dimensión prevista (Tabla 3). Observándose además, en ambos modelos, intercorrelaciones de bajas a moderadas entre los factores evidenciando una ade-




confirmatorios, en ambos modelos, alcanzan valores de consistencia interna iguales o por encima de .70; evidenciando una consistencia interna adecuada para este tipo de subescalas (Tabla 4).

\begin{tabular}{|c|c|c|c|c|}
\hline Factor & \multicolumn{2}{|c|}{ PSDQ-SMA } & \multicolumn{2}{|c|}{ PSDQ-SMB } \\
\hline & & & & \\
\hline Actividad física & .885 & .883 & .867 & .876 \\
\hline Apariencia & .870 & .870 & .827 & .827 \\
\hline Grasa corporal & .876 & .875 & .876 & .875 \\
\hline Coordinación & .861 & .856 & .834 & .828 \\
\hline Resistencia & .839 & .839 & .810 & .806 \\
\hline Flexibilidad & .878 & .873 & .862 & .860 \\
\hline Salud & .773 & .767 & .706 & .700 \\
\hline Competencia deportiva & .901 & .844 & .898 & .844 \\
\hline Fuerza & .855 & .857 & .766 & .755 \\
\hline Autoconcepto físico general & .906 & .906 & - & - \\
\hline Autoestima & .716 & .727 & - & - \\
\hline
\end{tabular}

\section{Discusión y Conclusiones}

De los resultados mostrados, de su análisis y tomando en cuenta que el objetivo principal de este estudio fue el de examinar la estructura interna de la versión corta del Physical Self Description Questionnaire (PSDQ-SM), se pueden obtener las siguientes conclusiones:

1) Los análisis factoriales confirmatorios realizados apoyan la estructura factorial de nueve factores (actividad física, apariencia, grasa corporal, coordinación, resistencia, flexibilidad, salud, competencia deportiva y fuerza), donde los factores así obtenidos presentan saturaciones factoriales estandarizadas adecuadas, saturaciones que, en general, se corresponden con la estructura propuesta para el cuestionario por Marsh et al. (2010); no obstante la necesidad de eliminar dos factores y nueve de los ítems. Además, los factores correlacionan entre sí de forma positiva y estadísticamente significativa lo cual muestra que a medida que mejora la percepción del autoconcepto físico en alguna de las dimensiones, también mejora en las otras.

2) Todos los factores evidenciaron una consistencia interna aceptable; a pesar del número reducido de ítems en cada uno de ellos.

El autoconcepto se forma por la percepción personal de las características de la persona como sobre peso, bajo peso o peso normal (Baena, Vega \& Ramírez, 2018), así como insatisfacción corporal y hábitos saludables que se adquieren desde edades tempranas (Longmuiremail, Colley, Wherley \& Tremblay, 2014), es de suma importancia contar con herramientas que apoyen en la medición del autoconcepto físico. En síntesis, el análisis de las propiedades psicométricas del cuestionario PSDQ-SM ha mostrado que una estructura de nueve factores, de acuerdo con los requisitos psicométricos establecidos, es válido y confiable. La estructura de nueve factores ha mostrado adecuados indicadores de ajuste y de validez. Contar con instrumentos con estas características son de suma importancia

Cuando menos dos limitaciones están presentes en este trabajo. La primera es que los participantes son solo estudiantes, lo que supone una amenaza para la posibilidad de generalizar estos resultados. Ampliar la muestra (agregando por ejemplo adolescentes que no sean estudiantes) es un área de trabajo de cara al futuro. La segunda limitación proviene del propio instrumento de medición, que se basa en el autoinforme y que por ello puede contener los sesgos que se derivan de la deseabilidad social.

Asimismo, en investigaciones futuras, es indispensable comprobar si el cuestionario resulta útil para predecir, por ejemplo, la insatisfacción corporal y problemas relacionados con conductas alimentarias.

\section{Agradecimientos}

La Secretaría de Educación Pública-Subsecretaría de Educación Superior-Dirección de Superación AcadémicaPrograma para el Desarrollo Profesional Docente (DE-13 6894) financió este estudio.

\section{Referencias}

Abalo, J., Lévy, J., Rial, A. \& Varela, J. (2006). Invarianza factorial con muestras múltiples. En J. Lévy (Ed.), Modelización con Estructuras de Covarianzas en Ciencias Sociales (pp. 259-278). Madrid: Netbiblo.

Arbuckle, J. R. (2012). AMOS users guide version 21.0. Chicago, IL: Marketing Department, SPSS Incorporated.

Baena, A. C. M., Vega, D. M. \& Ramírez, J. V. (2018). Factores predictores de la actividad física en escolares españoles de acuerdo a su estado de peso. Retos: nuevas tendencias en educación física, deporte y recreación (33), 7480.

Bailey,A., Hetrick, S., Rosenbaum, S., Purcell, R. \& Parker, A. (2018). Treating depression with physical activity in adolescents and young adults: a systematic review and meta-analysis of randomised controlled trials. Psychological medicine, 48(7), 1068-1083.

Blanco, J., Blanco, H., Viciana, J., \& Zueck, C. (2015). Psychometric properties of the physical self-concept questionnaire with mexican university students. Psychological reports, 116(2), 422-437.

Byrne, B. M. (2010). Structural Equation Modeling With AMOS: Basic Concepts, Applications, and Programming. New York, NY: Routledge.

Delgado-Floody, P., Carter-Thuillier, B., Jerez-Mayorga, D., Cofré-Lizama, A., \& Martínez-Salazar, C. (2018). Relación entre sobrepeso, obesidad y niveles de autoestima en escolares (Relation between overweight, obesity, and self-esteem levels in schoolchildren). Retos, (35), 67-70.

Elosua, P. \& Zumbo, B. D. (2008). Coeficientes de fiabilidad para escalas de respuesta categórica ordenadas. Psicothema, 20(4), 896-901.

ENSANUT. (2016). Encuesta Nacional de Salud y Nutrición de Medio Camino 2016 Informe Final de Resultados. Rcuperado de http://oment.uanl.mx/wp-content/ uploads/2016/12/ensanut_mc_2016-310oct.pdf.

Fernández, J. G., Contreras, O. R., García, L. M. \& González, S. (2010). Physical Self-concept depending on the kind of physical activity practised and motivation to it. Revista latinoamericana de psicología, 42(2), 251-263.

Fernández-Bustos, J.-G., González-Martí, I., Contreras, O. \& Cueva, R. (2015). Relación entre imagen corporal y autoconcepto físico en mujeres adolescentes. Revista latinoamericana de psicología, 47(1), 25-33.

Gelabert, E., García-Esteve, L., Martín-Santos, R., Gutiérrez, F., Torres, A. \& Subirà, S. (2011). Psychometric properties 
of the Spanish version of the Frost Multidimensional Perfectionism Scale in women. Psicothema, 23(1), 133139.

Grao-Cruces, A., Fernández-Martínez,A. \& Nuviala, A. (2016). Asociación entre condición física y autoconcepto físico en estudiantes españoles de 12-16 años. Revista latinoamericana de psicología, 49(2), 128-136. doi: http:// dx.doi.org/10.1016/j.rlp.2016.09.002

Kyle, T. L., Mendo, A. H., Garrido, R. E. R., \& Sánchez, V. M. (2016). Efectos de la actividad física en el autoconcepto y la autoeficacia en preadolescentes. Retos: nuevas tendencias en educación física, deporte y recreación, (29), 61-65.

Longmuiremail, P., Colley, R., Wherley, V. \& Tremblay, M. (2014). Risks and benefits of childhood physical activity. The lancet Diabets Endocrinologye Psychology, 2(11), 861-862. doi: 10.1016/S2213-8587(14)70221-9

Marsh, H. W. (1996). Construct validity of Physical SelfDescription Questionnaire responses: Relations to external criteria. Journal of sport and Exercise Psychology, 18(2), 111-131.

Marsh, H. W., Martin, A. J. \& Jackson, S. (2010). Introducing a Short Version of the Physical Self Description Questionnaire: new strategies, short-form evaluative criteria, and applications of factor analyses. Journal of Sport \& Exercise Psychology, 32(4), 438-482.

Marsh, H. W., Richards, G. E., Johnson, S., Roche, L. \& Tremayne, P. (1994). Physical Self-Description Questionnaire: Psychometric properties and a miiltitraitmeltimethod analysis of relations to existing instruments. Journal of sport and Exercise Psychology, 16(3), 270305.

Martínez, F. D. M., \& González-Hernández, J. (2018). Práctica de actividad física, conducta prosocial y autoconcepto en adolescentes: conexiones en el contexto escolar. Electronic Journal of Research in Education Psychology, 16(46), 555-577.

McMichan, L., Gibson, A.-M. \& Rowe, D. A. (2018). Classroom-Based Physical Activity and Sedentary Behavior Interventions in Adolescents: A Systematic Review and Meta-Analysis. Journal of Physical Activity and Health, 15(5), 383-393.

Montero, I. \& León, O. (2005). Sistema de clasificación del método en los informes de investigación en Psicología. International Journal of Clinical and Health Psychology, 5, 115-127.

Moreno, J. A., Cervelló, E. M. \& Moreno, R. (2008). Importancia de la práctica físico-deportiva y del género en el autoconcepto físico de los 9 a los 23 años. International Journal of Clinical and Health Psychology, 8(1).

Murgui, S., García, C. \& García, Á. (2016). Efecto de la práctica deportiva en la relación entre las habilidades motoras, el autoconcepto físico y el autoconcepto multidimensional. Revista de psicología del deporte, 25(1), 19-25.

Nunnally, J. C. \& Bernstein, I. H. (1995). Teoría Psicométrica. México: McGraw-Hill.

Organización Mundial de la Salud. (2018a). Obesidad y sobrepeso. Recuperado de http://www.who.int/es/newsroom/fact-sheets/detail/obesity-and-overweight.
Organización Mundial de la Salud. (2018b). Adolescentes: riesgos para la salud y soluciones. Recuperado de: https:/ /www.who.int/es/news-room/fact-sheets/detail/ adolescents-health-risks-and-solutions

Pakarinen, A., Parisod, H., Smed, J. \& Salanterä, S. (2017). Health game interventions to enhance physical activity self efficacy of children: a quantitative systematic review. Journal of advanced nursing, 73(4), 794-811.

Reigal, R.E., Videra, A., Parra, J. L. \& Juárez, R. (2012). Actividad físico deportiva, autoconcepto físico y bienestar psicológico en la adolescencia. RETOS. Nuevas tendencias en Educación Física, Deporte y Recreación. (22), 19-23. ISSN 1579-1726.

Revelle, W. \& Zinbarg, R. E. (2009). Coefficients alpha, beta, omega and the glb: comments on Sijtsma. Psychometrika, 74(1), 145-154. doi: 10.1007/s11336-008-9102-z

Rodríguez, A., González-Fernández, Ó. \& Goñi, A. (2013). Sources of perceived sociocultural pressure on physical self-concept. Psicothema, 25(2), 192-198. doi: 10.7334/ psicothema2012.229

Rodríguez, M. N. \& Ruiz, M. A. (2008). Atenuación de la asimetría y de la curtosis de las puntuaciones observadas mediante transformaciones de variables: Incidencia sobre la estructura factorial. Psicológica, 29, 205-227.

Rosa, A. García, E., \& Carrillo, P. J. (2019). Actividad física, condición física y autoconcepto en escolares de 8 a 12 años. Retos: nuevas tendencias en educación física, deporte y recreación, (35), 236-241.

Sijtsma, K. (2009). On the use, the misuse, and the very limited usefulness of Cronbach's alpha. Psychometrika, 74(1), 107-120. doi: 10.1007/s11336-008-9101-0

Thompson, B. (2004). Exploratory and Confirmatory Factor Analysis. Understanding concepts and applications. Washington, D C: American Psychological Association.

Yáñez, R., Barraza, F. \& Mahecha, S. (2016). Actividad física, rendimiento académico y autoconcepto físico en adolescentes de Quintero, Chile. Educación Física y Ciencia, 18(2), 00-00.

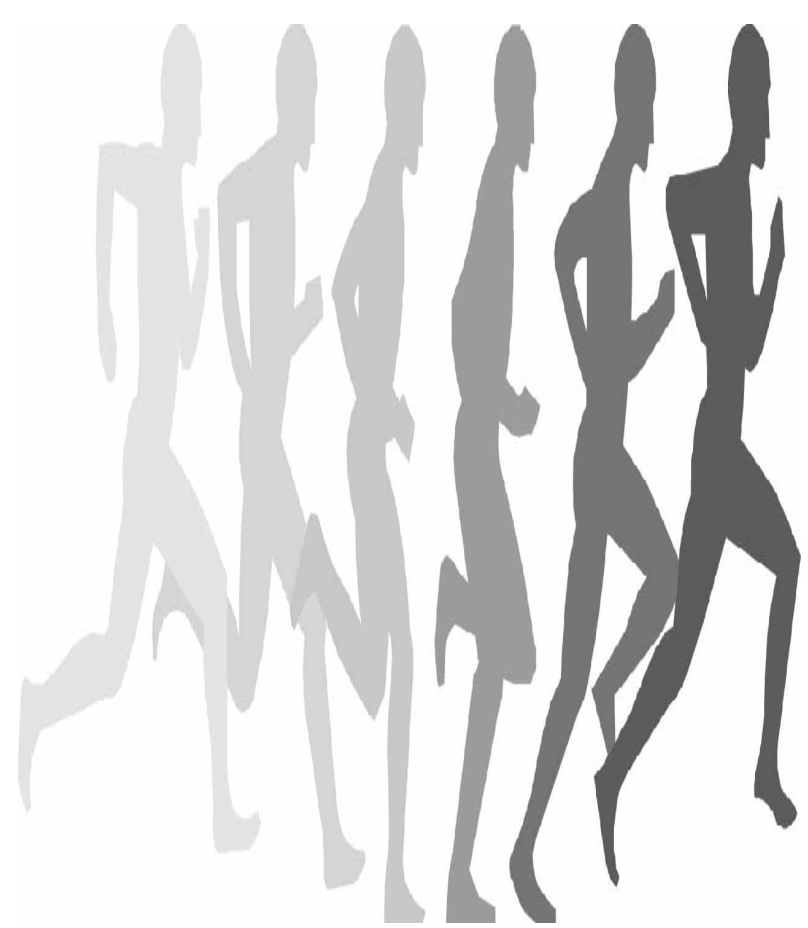

